\title{
man \\ Precipitant-Free Crystallization of Lysozyme and Glucose Isomerase by Drying
}

\author{
Yoshihisa Suzuki ${ }^{1, *(D)}$, Shiori Fujiwara ${ }^{2}$, Shoko Ueta ${ }^{1}$ and Takashi Sakai ${ }^{3}$ \\ 1 Graduate School of Technology, Industrial and Social Sciences, Tokushima University, 2-1 Minamijosanjima, \\ Tokushima 770-8506, Japan; shoko.ueta@tokushima-u.ac.jp \\ 2 Graduate School of Advanced Technology and Science and Technology, Tokushima University, \\ 2-1 Minamijosanjima, Tokushima 770-8506, Japan; c612133008@tokushima-u.ac.jp \\ 3 Institute for Health Sciences, Tokushima Bunri University, 180 Houji, Nishihama, Yamashiro, \\ Tokushima 770-8514, Japan; tsakai@tks.bunri-u.ac.jp \\ * Correspondence: yoshis@tokushima-u.ac.jp; Tel.: +81-88-656-7415
}

check for updates

Citation: Suzuki, Y.; Fujiwara, S.; Ueta, S.; Sakai, T. Precipitant-Free Crystallization of Lysozyme and Glucose Isomerase by Drying. Crystals 2022, 12, 129. https:// doi.org/10.3390/cryst12020129

Academic Editors: Vladimir Timofeev, Hiroaki Tanaka, Yuri Pisarevsky,

Margarita Marchenkova and

Abel Moreno

Received: 1 December 2021

Accepted: 15 January 2022

Published: 18 January 2022

Publisher's Note: MDPI stays neutral with regard to jurisdictional claims in published maps and institutional affiliations.

Copyright: (C) 2022 by the authors. Licensee MDPI, Basel, Switzerland. This article is an open access article distributed under the terms and conditions of the Creative Commons Attribution (CC BY) license (https:// creativecommons.org/licenses/by/ $4.0 /)$.

\begin{abstract}
Protein crystallization is usually conducted by using precipitants, although the "salting-out" phenomenon is still unclear and complex. Moreover, the addition of precipitants sometimes results in irreversible disordered precipitation of protein molecules. Although precipitant-free lysozyme crystals obtained by centrifugal concentration showed significant changes in three-dimensional structure compared to the structure of salted-out crystals, it was rather difficult to mount crystals from a viscous dense liquid phase after centrifugal concentration, and the quality of the crystals often deteriorated during the mounting process. Here we present novel precipitant-free crystallization methods, which were effective for lysozyme and glucose isomerase. Tetragonal lysozyme crystals were successfully crystallized in a glass capillary simply by drying highly concentrated lysozyme solution in the presence of $0.01 \mathrm{M}$ hydrochloric acid without using any precipitants. Glucose isomerase dissolved in ultra-pure water was also successfully crystallized in hanging drops by drying highly concentrated solution under low-humidity conditions. Oscillation images of the obtained crystals were safely collected without handling; they clearly indicated the crystals had a tetragonal form for lysozyme and an orthorhombic form for glucose isomerase, and their lattice parameters are similar to those of previously reported crystals obtained by salting-out methods.
\end{abstract}

Keywords: precipitant-free; drying; highly concentrated solution; oscillation image

\section{Introduction}

High quality protein crystals that can be analyzed at high resolution $(<1.5 \AA)$ are prerequisites for structure-based drug design, which is defined as a method to optimize the potency of a drug using the precise structural information of a target protein [1]. As the protein data bank (PDB) shows that 15,183 structures of protein molecules deposited until 22 December 2021 show their resolution limit higher than $1.5 \AA$ [2], many researchers have crystallized huge number of proteins using various methods (dialysis, vapor diffusion, batch, gel-mediated, and so forth) [3] and conditions (under high magnetic field [4], electric field [5], flow [6], high pressure [7], microgravity [8], and so forth). In spite of a wide variety of methods and conditions, most of the proteins were commonly crystallized using precipitants. Although almost all structures in PDB are obtained using crystals grown with large amounts of precipitants, such conditions are quite different from those in general living organisms; the positions of important molecules of the three-dimensional structures of protein molecules would sometimes differ by more than $1.5 \AA$ from those of native structures. Thus, we tried to crystallize lysozyme simply by centrifugal concentration without using any precipitants [9]. The obtained structure clearly showed that lysozyme loses a sodium cation and changes the position of the hydroxyl oxygen of Ser72 by more 
than $1.5 \AA$ from that of the structure (PDB ID 2ZQ3) of crystals obtained using sodium chloride as a precipitant.

However, in our previous study, as crystals were nucleated in a highly concentrated and viscous liquid phase after liquid-liquid phase separation by centrifugal concentration processes, it was always difficult to mount crystals using cryo-loops without a deterioration of their quality [9]. Moreover, Masuda et al. revealed that the positions of water molecules adjacent to an activation site of proteinase $\mathrm{K}$ at ambient temperature significantly differed from those of cryo-cooled structures [10]. They collected the data at ambient temperature using femtosecond X-ray pulses from the SPring-8 Angstrom Compact Free Electron Laser (Hyogo, Japan). More recently Bradford et al. found the conformational changes in response to temperature in the T4 lysozyme L99A cavity by single crystal synchrotron X-ray crystallography [11]. They collected diffraction data at both ambient temperature (278 K) and cryogenic temperature $(100 \mathrm{~K})$ using synchrotron X-ray diffraction at the Advanced Photon Source (Lemont, IL, USA) beamline ID-24. If we can collect diffraction data of high-quality precipitant-free crystals without touching them at ambient temperature, we could collect complete and high-quality diffraction data sets with truly native structures. The drying process in glass capillary or in hanging-drops on glass slides under adequate humidity conditions would be favorable, as we could mount crystals without touching them for both processes. Actually, we have already developed a handling-free mounting method using glass capillaries for X-ray crystallography of protein crystals at ambient temperature [12]. Although Ghatak et al. showed that lysozyme and proteinase K seemed to be crystallized by the evaporation of water at $20{ }^{\circ} \mathrm{C}$, they argued that the crystals were obtained due to the effects of an incision on plasma oxidized polydimethylsiloxane substrate; they claimed that crystals were not obtained by concentration of proteins by evaporation of water. Moreover, they did not show the diffraction data of the crystals [13].

In this study, we showed the results of the complete drying process at first; if a slow evaporation process could maintain the ordered structure of the crystal, the evaporation process would be effective for controlling the nucleation and growth processes of protein crystals. We found clear diffraction spots from completely dried crystals of hen eggwhite lysozyme (HEWL); even in dried condition, regular arrays of lysozyme sufficiently remained. We then showed that HEWL and glucose isomerase (GI) could be crystallized simply by drying; HEWL was crystallized by the slow evaporation of water in glass capillaries, and GI was crystallized by slow evaporation of water in hanging drops. All obtained crystals were characterized by using oscillation images of X-ray diffraction.

\section{Materials and Methods}

HEWL (Seikagaku, six times recrystallized) and GI (Nagase ChemteX, Spezyme GIpf) were used as model proteins without further purification. We tried to concentrate the HEWL in $0.01 \mathrm{M}$ aqueous hydrochloric acid $(\mathrm{HCl})$ solution, since the solubility of a protein crystal without precipitants is known to be very high [14] and since irreversible aggregation would be effectively inhibited at low $\mathrm{pH}$ owing to the repulsive interactions between positively charged surfaces of HEWL molecules. We first desalted and concentrated the HEWL solution in $0.01 \mathrm{M} \mathrm{HCl}$ using a centrifugal filter unit (Millipore, Ultracel-3K) five times, and then evaporated water from the concentrated solution slowly in a glass capillary (Hampton, HR6-116) through caps which are made of silicone-type adhesive at both ends of the capillary. As for GI, we desalted and concentrated it in ultra-pure water (Millipore, Simplicity UV, resistivity greater than $18 \mathrm{M} \Omega \mathrm{cm}$ ) by centrifugal concentration. A $5 \mu \mathrm{L}$ drop of concentrated GI solution was layered on a $15 \mathrm{~mm}$ diameter coverslip (Matsunami, Micro cover glass), returned, and set on the rim of one well of a 96-well plate (As one, Violamo 96 well test plate F-bottom). The rim was greased with Vaseline to make a seal, and $30 \mu \mathrm{L}$ saturated aqueous sodium chloride $(\mathrm{NaCl})$ solution was used as a reservoir solution at the bottom of the well. The quality of the obtained crystals was characterized by oscillation images using in-house X-ray sources (Rigaku, RAXIS-VII and RAXIS-RAPID II) and synchrotron X-ray radiation facilities (SPring-8, BL44XU). 


\section{Results}

\subsection{Completely Dried, Transparent, and Bulk HEWL Crystals}

This study was started from an accidental discovery of the ordered structure of a dried and transparent bulk solid of lysozyme. For another purpose, we added dissolved and highly concentrated (up to $300 \mathrm{mg} \mathrm{mL}^{-1}$ ) HEWL to $0.01 \mathrm{M}$ aqueous hydrochloric acid solution in a glass capillary with both ends silicone-capped. After several weeks, we found a completely dried, hard, and transparent bulk solid in the capillary (Figure 1a). Oscillation images (oscillation angle is $1^{\circ}$, and exposure time is $60 \mathrm{~s}$ ) were taken using an in-house X-ray source (Rigaku, R-AXIS VII output $2.0 \mathrm{~kW}$ ) as a trial at ambient temperature. Clear diffraction spots were confirmed. From the three images $\left(0^{\circ}, 60^{\circ}, 120^{\circ}\right)$, the crystal structure of the tetragonal form (unit cell dimensions: $a=b=73.277 \AA$ and $c=31.777 \AA$ ) was determined. This result clearly indicates that a very large single crystal existed in the capillary before complete drying: drying processes are effective for protein crystallization. Moreover, even when completely dried, solid protein keeps its ordered structure. Although the number of spots is limited possibly due to the loss of the water around HEWL molecules and the subsequent disordering of the precise structure of side chain molecules that remain in the presence of hydrated water molecules, this bulk solid protein would keep a regular array of nanometer-sized pores, and could be used as a novel class of nanoporous materials [15].

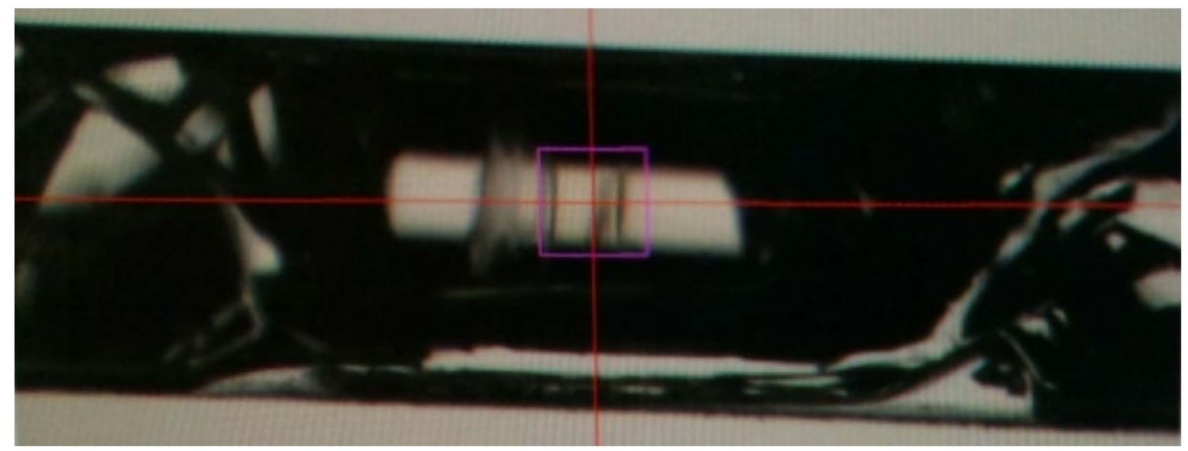

(a)

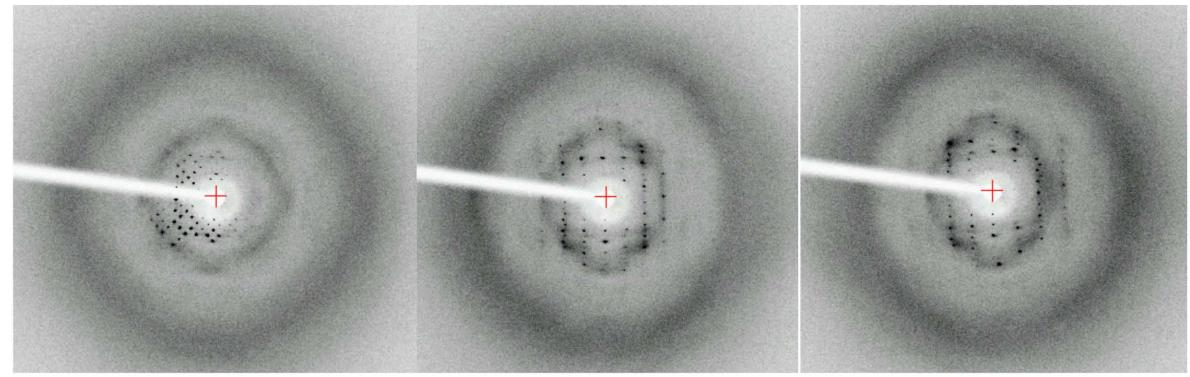

(b)

Figure 1. (a) A completely dried and transparent bulk object in a glass capillary (inner diameter, $0.7 \mathrm{~mm}$ ). (b) Oscillation images of the object (oscillation angle $1^{\circ}$, exposure time $60 \mathrm{~s}$ ).

The stability of HEWL in a $0.01 \mathrm{M} \mathrm{HCl}$ solution would be good, since we did not find any irreversible precipitates throughout the experiments in this study. The $\mathrm{pH}$ of $239 \mathrm{mg} \mathrm{mL}^{-1} \mathrm{HEWL}$ in $0.01 \mathrm{M} \mathrm{HCl}$ solution was 3.36 in this study. Venkataramani et al. measured the melting (unfolding) temperatures $T_{\mathrm{m}}$ of lysozyme using FTIR spectroscopy [16]. From the results, the $T_{\mathrm{m}}$ of lysozyme at $\mathrm{pH}=3.0$ was $68{ }^{\circ} \mathrm{C}$. We did experiments at room temperature at the highest, thus sufficiently stable conditions would be realized. 


\subsection{Raw HEWL Crystals Obtained during Drying Processes in Glass Capillaries}

We tried to grow raw crystals of HEWL simply by drying processes. Slow evaporation and limited nucleation of HEWL crystals were achieved using the concentrated HEWL solution filled in silicone-capped glass capillaries prepared as described in Materials and Methods. After several days, some capillaries started to show the nucleation and growth of glittering crystals along the curved interior wall of the glass capillary (Figure 2). Figure 3 shows an oscillation image (oscillation angle $1^{\circ}$, exposure time $300 \mathrm{~s}$ ) taken using an inhouse X-ray source (Rigaku, R-AXIS RAPID II, output $1.5 \mathrm{~kW}$ ) at ambient temperature. Clear diffraction spots were confirmed. From six images, the lattice parameters were calculated to be $a=b=78.4 \AA, c=38.0 \AA$ (tetragonal form, $\mathrm{P} 4{ }_{3} 2{ }_{1} 2$ ). These values are similar to those of previously obtained data $(a=b=78.54 \AA, c=37.77 \AA$ (PDB ID $=193 \mathrm{~L})$ ). However, the three-dimensional molecular structure of a specific site (for instance, the $\mathrm{Na}^{+}$ site as reported in [9]) could be significantly changed. Although to confirm the significance we still have to collect sufficient diffraction data, we confirm for the first time the efficacy of simple drying for high-quality protein crystallization.

\subsection{GI Crystals Obtained Simply by Drying in Hanging Drops}

A highly concentrated ( $\left.200 \mathrm{mg} \mathrm{mL}^{-1}\right)$ GI drop showed nucleation at the rim of the drop immediately after setting the coverslip with the drop on the well with $30 \mu \mathrm{L}$ saturated aqueous $\mathrm{NaCl}$ solution as a reservoir. After a few hours, crystals grew inward into the drop, as shown in Figure 4. The grains of the GI crystals grew, and geometrical selection of the grains occurred [17]. Then, we lifted the coverslip off the well with $\mathrm{NaCl}$ solution, and reset it on the other well without a reservoir to slow the inward growth. Synchrotron X-ray oscillation images were collected by mounting the coverslip, which was covered with a polyimide sheet (DuPont, Kapton $50 \mathrm{H}-\mathrm{A} 4$, thickness $12.5 \mu \mathrm{m}$ ) cut into a circular shape (diameter was $15 \mathrm{~mm}$ ) and a silicone rubber sheet spacer (Togawa Rubber, K-125, thickness was $1 \mathrm{~mm}$ ) cut with a doughnut-shape (inner diameter $8 \mathrm{~mm}$, outer diameter $15 \mathrm{~mm}$ ) to avoid further drying of the drop, on a synchrotron X-ray beamline (SPring-8, BL44XU) at ambient temperature. As a result of the selection of the number of grains, we could easily select large grains to collect oscillation images, the same as with single crystals. Data were collected at room temperature. Figure 5 shows an oscillation image (oscillation angle $0.5^{\circ}$, exposure time $1 \mathrm{~s}$, attenuator $\mathrm{Al} 2.1 \mathrm{~mm}$, camera distance $150 \mathrm{~mm}$, wavelength $0.9 \AA)$. Clear diffraction spots can be observed up to the resolution limit $(1.50 \AA)$ shown as part of a border curve below the hatched area (Figure 5a). Using 326 oscillation images, the lattice parameters were calculated to be $a=94.08 \AA, b=99.32 \AA, c=103.13 \AA$ (orthorhombic form, I222). These values are similar to previously obtained data $(a=93.88 \AA, b=99.68 \AA$, $c=102.9 \AA$ (PDB ID = 1XIB)). As described in Section 3.2, we confirmed the availability of simple drying for high-quality protein crystallization.

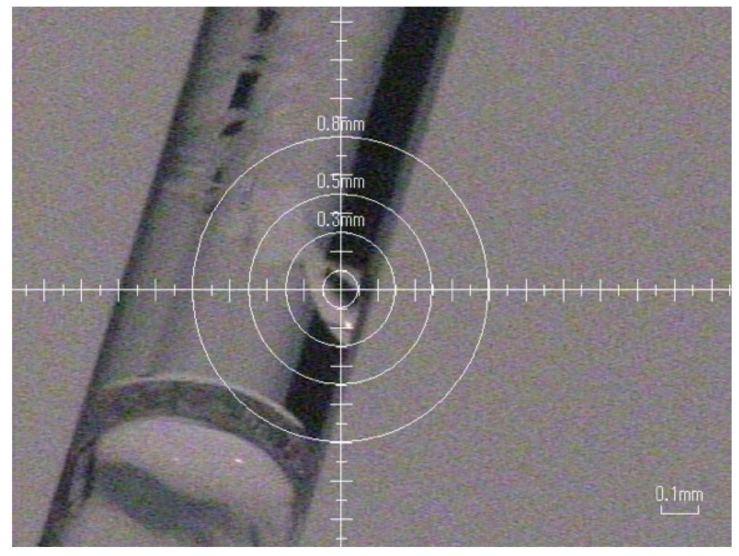

Figure 2. Crystals grown along the curved inside wall of a glass capillary. The crystal at the center of the target is used for data collection. 


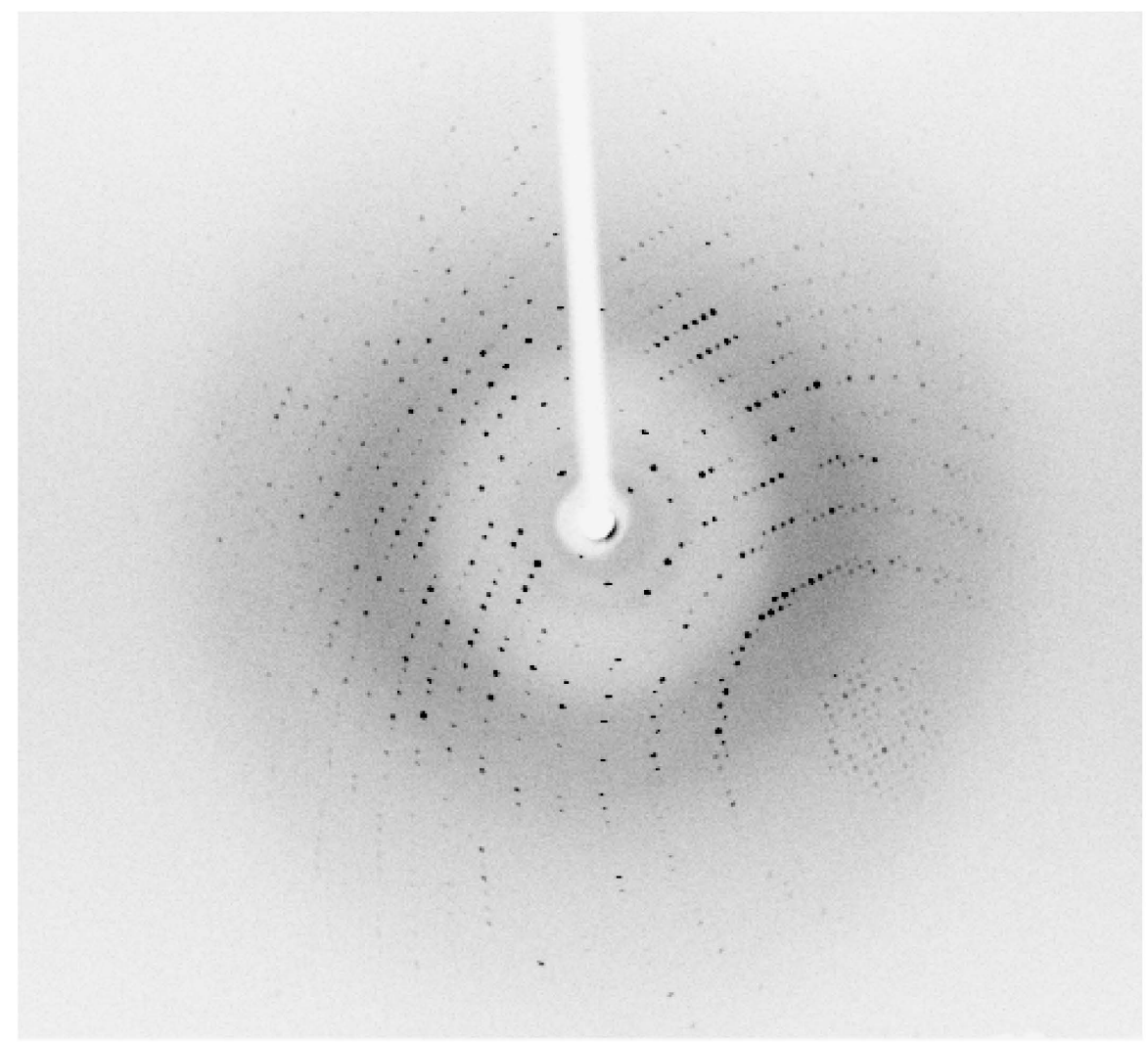

Figure 3. An oscillation image (oscillation angle $1^{\circ}$, exposure time $300 \mathrm{~s}$ ) of the crystal shown in Figure 2. Clear diffraction spots are confirmed.

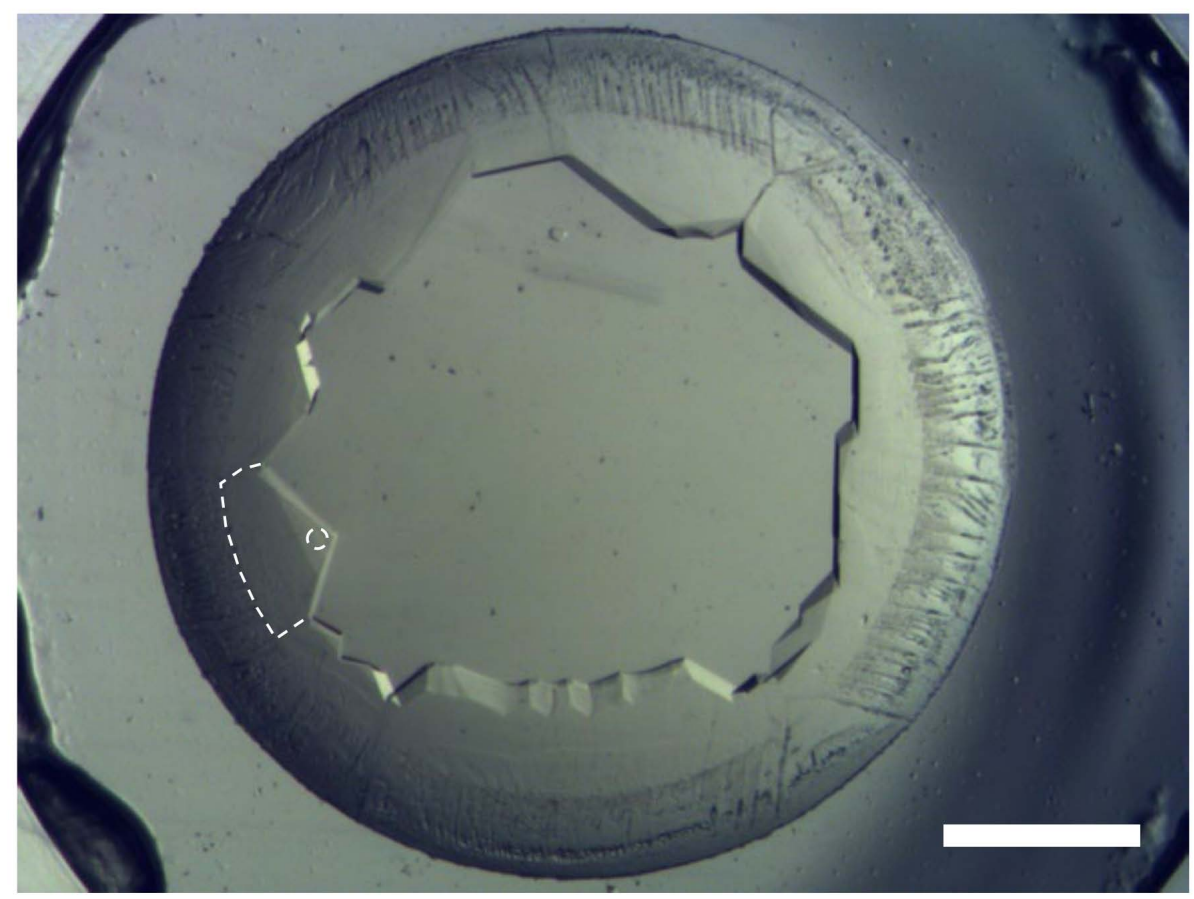

Figure 4. Crystals nucleated at the rim of a drop and grew inward. Geometrical selection occurred during the inward growth. Scale bar represents $1 \mathrm{~mm}$. The broken line and circle show the boundary of a grain used for data collection and the point of irradiation, respectively. 

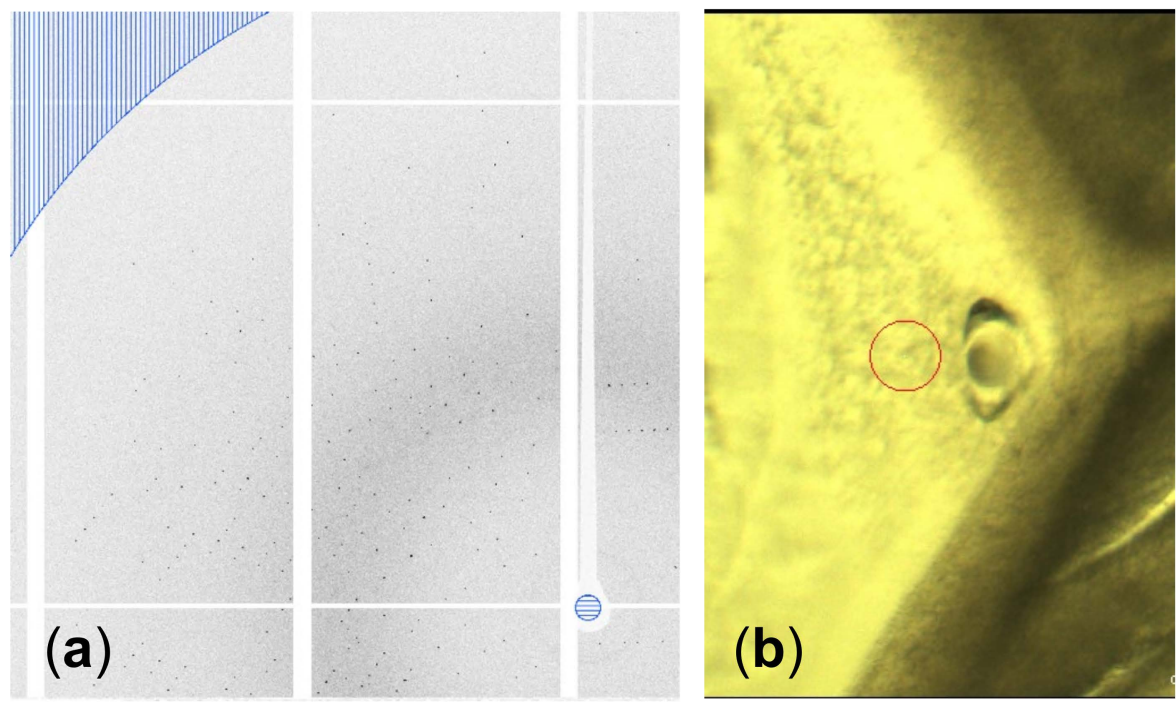

Figure 5. (a) Part of an oscillation image. The resolution limit, shown as a border curve below the hatched area, is $1.50 \AA$. (b) Part of a grain shown in Figure 4. A hole formed after data collection due to the dissolution caused by the temperature increase from the irradiation of the synchrotron X-ray. The red circle indicates the beam size used for the data collection (diameter $50 \mu \mathrm{m}$ ).

\section{Discussion}

The use of a precipitant-free method becomes important when the structure under a precipitant-free condition differs significantly from that with copious amounts of precipitants. At this stage, we have not collected high-quality diffraction data sets to judge the importance of our drying method, as we have not controlled nucleation and growth rates well; for instance, the overlapping of diffraction spots from other crystals that grew in different directions or the suppression of the diffraction intensity from smaller crystals caused incomplete data sets when many crystals nucleated under rapid and uncontrolled drying conditions.

On the other hand, although structure data has not been deposited in the PDB, the electron density map on the hydroxyl oxygen of Ser 72 seems to correspond to the backbone structure (Figure S1), the sodium cation near the Ser 72 residue of the HEWL seems to disappear owing to the precipitant-free condition, and the direction of the hydroxyl group seems to correspond to that of our previous precipitant-free HEWL structure [9]. The crystal used for the analysis was obtained by drying in a polyimide tube (Hagitec Co., Ltd., Yotsukaido, Chiba, Japan, PIT-LS-3.0), as shown in Supplementary Materials (Table S1 and Figure S1). To collect more precise data sets for the discussion of changes in threedimensional structures in precipitant-free crystals, we have to make the drying method more sophisticated by controlling the humidity precisely. Actually, in Section 3.3 we keep the humidity constant $(75 \%)$ by using saturated aqueous $\mathrm{NaCl}$ solution in the well to dry a hanging-drop, whereas, for more precise humidity control, we have to use various saturated solutions as reservoirs [18]. In a future work, precise, quantitative and active humidity control will be needed for the precise control of the nucleation and growth of crystals. In addition, we will apply these drying techniques for the other proteins.

Supplementary Materials: The following supporting information can be downloaded at: https:// www.mdpi.com/article/10.3390/cryst12020129/s1, Supplementary text: Preliminary three-dimensional structure of a lysozyme molecule from crystals obtained simply by drying; Figure S1: Alignment of our preliminary HEWL structure with the structure of 193L; Table S1: Data collection statistics of the HEWL crystal obtained by drying; Figure1_index.pdf and Figure3_index.pdf: Log files for indexing diffraction spots; Figure5_data_analyses.pdf: A log file for data analyses for Figure 5; HEWL_FigureS1.mmcif. 
Author Contributions: Conceptualization, Y.S.; methodology, Y.S., S.U. and T.S.; investigation, Y.S. and S.F.; writing - original draft preparation, Y.S.; writing-review and editing, Y.S.; funding acquisition, Y.S. All authors have read and agreed to the published version of the manuscript.

Funding: This study was supported by JSPS KAKENHI Grant nos. 24656016, 26390054, 15K05668, 16K05470, 18K04960, 20K03782, and 21K04908. This study was partly supported by a Grant for the Joint Research Program of the Institute of Low Temperature Science, Hokkaido University (20G029 and 21G021).

\section{Institutional Review Board Statement: Not applicable.}

Informed Consent Statement: Not applicable.

Data Availability Statement: Data is contained within the article and Supplementary Materials. The $\log$ files for indexing diffraction spots in Figures 1 and 3 are available as Figure1_index.pdf and Figure3_index.pdf. A log file for data analyses for Figure 5 is available as Figure5_data_analyses.pdf. An mmCIF file for Figure S1 is also available as HEWL_FigureS1.mmcif.

Acknowledgments: This work was performed using a synchrotron beamline BL44XU at SPring8 under the Collaborative Research Program of Institute for Protein Research, Osaka University. Diffraction data were collected at the Osaka University beamline BL44XU at SPring-8 (Harima, Japan) (Proposal Nos. 2017A6726, 2017B6726, 2018A6826, 2018B6826, 2019A6925, 2019B6925, 2020A6523, 2020B6523, 2021A6622, and 2021B6622). Y.S. thanks Eiki Yamashita, Masato Yoshimura, and Keisuke Sakurai of SPring-8/BL44XU for data collection in the collaborative study program. Y.S. also thanks Gen Sazaki of Hokkaido University, Masahide Sato of Kanazawa University, and Hiroyasu Katsuno for valuable discussion in the Joint Research Program of the Institute of Low Temperature Science, Hokkaido University.

Conflicts of Interest: The authors declare no conflict of interest.

\section{References}

1. Davis, A.M.; Teague, S.J.; Kleywegt, G.J. Application and limitations of x-ray crystallographic data in structure-based ligand and drug design. Angew. Chem. Int. Ed. 2003, 42, 2718-2736. [CrossRef] [PubMed]

2. A Structural View of Biology. Available online: https://www.rcsb.org (accessed on 22 December 2021).

3. Ducruix, A.; Giegé, R. Crystallization of Nucleic Acids and Proteins, 2nd ed.; Oxford University Press: New York, NY, USA, 1999; pp. 121-176.

4. Sazaki, G.; Yoshida, E.; Komatsu, H.; Nakada, T.; Miyashita, S.; Watanabe, K. Effects of a magnetic field on the nucleation and growth of protein crystals. J. Cryst. Growth 1997, 173, 231-234. [CrossRef]

5. Taleb, M.; Didierjean, C.; Jelsch, C.; Mangeout, J.P.; Capelle, B.; Aubry, A. Crystallization of proteins under an extenal electric field. J. Cryst. Growth 1999, 200, 575-582. [CrossRef]

6. Kadowaki, A.; Yoshizaki, I.; Adachi, S.; Komatsu, H.; Odawara, O.; Yoda, S. Effects of forced solution flow on protein-crystal quality and growth process. Cryst. Growth Des. 2006, 6, 2398-2403. [CrossRef]

7. Visuri, K.; Kaipainen, E.; Kivimaki, J.; Niemi, H.; Leisola, M.; Palosaari, S. A new method for protein crystallization using high pressure. Bio/Technology 1990, 8, 547-5491. [CrossRef] [PubMed]

8. Suzuki, Y.; Tsukamoto, K.; Yoshizaki, I.; Miura, H.; Fujiwara, T. First direct observation of impurity effects on the growth rate of tetragoal lysozyme crystals under microgravity as measured by interferometry. Cryst. Growth Des. 2015, 15, 4787-4794. [CrossRef]

9. Suzuki, Y.; Tsuge, H.; Hondoh, H.; Kato, Y.; Uehara, Y.; Maita, N.; Hosokawa, K.; Ueta, S. Precipitant-free lysozyme crystals grown by centrifugal concentration reveal structural changes. Cryst. Growth Des. 2018, 18, 4226-4229. [CrossRef]

10. Masuda, T.; Suzuki, M.; Inoue, S.; Song, C.; Nakane, T.; Nanogo, E.; Tanaka, R.; Tono, K.; Joti, Y.; Kameshima, T.; et al. Atomic resolution structure of serine protease proteinase K at ambient temperature. Sci. Rep. 2017, 7, 45604. [CrossRef] [PubMed]

11. Bradford, S.Y.C.; Khoury, L.E.; Ge, Y.; Osato, M.; Mobley, D.L.; Fischer, M. Temperature artifacts in protein structures bias ligand-binding predictions. Chem. Sci. 2021, 12, 11275-11293. [CrossRef] [PubMed]

12. Suzuki, Y.; Torii, K.; Inaka, K.; Fujiwara, T.; Tanaka, H.; Arai, Y. A novel handling-free method of mounting single protein crystals for synchrotron structure analyses at room temperature. Rev. Sci. Instrum. 2019, 90, 054101. [CrossRef] [PubMed]

13. Ghatak, A.S.; Rawal, G.; Ghatak, A. Precipitant-free crystallization of protein molecules induced by incision on substrate. Crystals 2017, 7, 245. [CrossRef]

14. Retailleau, P.; Ries-Kautt, M.; Ducruix, A. No salting-in of lysozyme chloride observed at low ionic strength over a large range of pH. Biophys. J. 1997, 73, 2156-2163. [CrossRef]

15. Vilenchik, L.Z.; Griffith, J.P.; St Clair, N.; Navia, M.A.; Margolin, A.L. Protein crystals as novel microporous materials. J. Am. Chem. Soc. 1998, 120, 4290-4294. [CrossRef] 
16. Venkataramani, S.; Truntzer, J.; Coleman, D.R. Thermal stability of high concentration lysozyme across varying pH: A Fourier Transform Infrared study. J. Pharm. Bioall. Sci. 2013, 5, 148-153. [CrossRef] [PubMed]

17. Suzuki, Y.; Sawada, T.; Tamura, K. Colloidal crystallization by a centrifugation method. J. Cryst. Growth 2011, 318, 780-783. [CrossRef]

18. International Critical Tables; McGraw-Hill: New York, NY, USA, 1926; Volume I, pp. 67-68. 